\title{
COLLOQUE COGNITIVA 85
}

Le colloque Cognitiva 85 , organisé sous l'égide du CESTA, et qui s'est déroulé du 4 au 7 juin dernier, avait pour sous-titre : « de l'Intelligence artificielle aux Bio-sciences ». On sait que l'Intelligence artificielle s'était dégagée de la cybernétique et ne se souciait plus d'assurer une quelconque analogie avec les mécanismes neuronaux. Récemment, les modèles néo-connexionnistes (réseaux d'automates capables d'apprentissage, de reconnaissance de formes, de mémoire associative), tout en restant indépendants de la neurobiologie, ont prétendu développer et tester la faisabilité d'hypothèses qui pourraient permettre aux neurobiologistes de modéliser certains aspects du fonctionnement du cerveau. Alors que l'Intelligence artificielle s'était tournée d'abord vers les psychologues, l'apport néo-connexionniste (néo-, parce que ces réseaux sont des héritiers des réseaux de neurones formels de $\mathrm{Mc}$ Culloch et Pitts) lui permet maintenant, via l'intégration de la Reconnaissance de Formes, de susciter l'intérêt des neurobiologistes. Le colloque Cognitiva s'est voulu le témoin de cette convergence entre des courants, jusqu'à il y a peu, séparés.

Un « Forum » avait pour but de proposer une lecture panoramique des différents courants de l'intelligence artificielle. Si les exposés consacrés aux différents programmes coordonnant les recherches en informatique et en IA, ou encore aux différentes applications des systèmes experts prenaient l'allure de catalogues, d'autres ont bien situé les différents langages de programmation (langages procéduraux classiques, langages de fonctions et de listes comme Lisp, langages logiques déclaratifs comme Prolog, langages orientés objets comme Smalltalk, où l'objet conjoint faits et méthodes pour opérer sur ce fait, etc.). De même, pour les problèmes d'adaptation des architectures des machines aux problèmes de l'IA (qui traite de relations entre symboles et non d'opérations sur des nombres, et donc a besoin, entre autres, d'une plus grande capacité de mémoire). Les recherches actuelles tendent à développer des architectures de traitement parallèle (opérations simultanées sur plusieurs données). On retrouve ici les problèmes des réseaux (bien qu'ils soient actuellement simulés sur des machines séquentielles). Le Forum s'est terminé par une table ronde philosophique où Hubert Dreyfus a repris ses critiques contre les systèmes experts comme simulation du comportement humain. Pour lui, un expert ne raisonne pas en appliquant des règles (si telle situation, alors telle action), mais en découvrant des analogies avec un 
des milliers de cas particuliers qu'il a mémorisés. Mais il n'a pas montré (et cela seul serait déterminant), qu'une application de règles était incapable de donner les mêmes résultats, c'est-à-dire de reconstruire ou simuler le processus de l'expert.

Le colloque proprement dit a permis l'expression des principaux courants de l'LA actuelle, une place importante étant réservée au néo-connexionnisme. Les systèmes experts ont été au centre des communications (cf. par exemple Laurière). Un système expert comprend une base de connaissance modulaire, à laquelle on peut donc ajouter des règles sans avoir à modifier toute son organisation, et un moteur d'inférences. Les connaissances sont celles d'un domaine spécialisé. Le problème se pose cependant de structurer la base de connaissances, de vérifier sa cohérence. On peut espérer aussi transférer la connaissance d'un système expert spécialisé - si elle est suffisamment formelle - dans d'autres domaines de spécialisation. Finalement, il faut aussi des systèmes experts pour diriger le dialogue avec l'expert humain et pour tester la cohérence de la base de connaissance (voire pour concevoir de nouveaux systèmes experts : conception assistée par ordinateur). C'est dans le domaine du diagnostic médical que les tentatives présentées étaient les plus nombreuses.

Citons encore des recherches plus fondamentales, en théorie formelle de l'apprentissage (identifié à la construction de théorèmes pour un sous-ensemble d'une classe d'objets) (Kodratoff, Sallantin). De nombreuses méthodes et algorithmes ont été proposés en reconnaissance des formes (étude des textures, des contours en optique, ou encore reconnaissance de la parole). Pour les réseaux, citons les communications de Hinton, qui oppose à la machine de von Neumann le concept de machine de Boltzmann (réseau d'automates à seuils, où l'on utilise la méthode du recuit simulé - on élève la " température " pour échapper aux minima purement locaux, puis on la rebaisse) ; Huberman (réseaux systoliques à plusieurs couches, simulant l'apprentissage pavlovien), Kohonen (mémoires associatives, qui recréent le contenu manquant à partir du reste du souvenir) ; Geman ; Fogelman. Les neurophysiologues ont témoigné de leur intérêt pour ces modèles comme de leur abstraction par rapport à la richesse des mécanismes neuronaux (Changeux, Imbert, Paillard, Bourguignon, Varela). S'il n'y a pas toujours eu dialogue entre ces multiples courants, le concert en tout cas était réussi. Le Cesta a publié en nombre limité les actes de ce colloque.

Pierre Livet. 